\title{
Methods and Guidelines for the Design and Development of Domestic Ubiquitous Computing Applications
}

\author{
Albrecht Schmidt \\ Fraunhofer IAIS and B-IT, University of Bonn \\ Germany \\ albrecht.schmidt@acm.org
}

\author{
Lucia Terrenghi \\ Media Informatics Group \\ University of Munich, Germany \\ lucia.terrennghi@ifi.lmu.de
}

\begin{abstract}
Bringing ubiquitous computing applications to home environments is a great challenge. In our research we investigate how applications can be conceived, designed, and implemented that fit into people's lives. In particular we describe our experiments on how methods of user centered design and participatory design can be appropriated to find users' requirements and design ideas for ubiquitous computing applications for the home. In particular we focus in the study reported on information presentation using display appliances. In a participatory design process, enhanced with technology probes, we discussed potential solutions for a specific home with 14 participants individually. Based on these individual solutions, fitting a single persona each, we specified the prototypes that would accommodate the user's needs and that are generic in its applicability. This allows drawing a first set of guidelines for the design of display appliances in the home environment.
\end{abstract}

\section{Introduction}

With the emergence of Ubicomp scenarios for everyday life, research has been addressing other environments besides the working place and the desktop environment, thus encountering new challenges in the elicitation and specification of user requirements [28]. In the last decade, the domestic environment has become the playground for "smart technology", in which scenarios of context awareness and automation of appliances have been tested in living laboratories [14], [16], [19], [21], [23]. However, the research has had little impact on ordinary homes, and the take-up of ubiquitous computing technologies in the home has been marginal so far.
Users' acceptance of the system, their privacy and trust concerns, together with controllability and learnability of the interface are main concerns that need to be addressed. This has suggested academic research to adopt ethnographic approaches to investigate this domain and look at its social patterns [1], [5], [20]. This research has its focus in understanding the users' needs but is in many cases very conservative with regard to technology. In our approach we acknowledge the importance of ethnographic research to elicit users' needs, but extend it by the in-situ introduction and discussion of new technology to gather design ideas.

To explore this approach further and to evaluate our methodology we investigated a specific application domain for ubiquitous computing in the home. We set out with the question: What display technologies are useful and desirable in a home environment? Our participatory design method included the analysis of how people use displays (mostly non-electronic) in their everyday lives. Starting from conventional displays, we investigate possible novel display artefacts in the domestic environment. We discussed with people potential ubiquitous computing display technologies in the concrete setting of their home environment.

The contribution of the paper is twofold:

- a method and approach for researching the potential use of ubiquitous computing technologies in the home;

- a number of design prototypes for domestic display appliances that have been created in a user centered design process.

\subsection{A taxonomy of domestic display artefacts}

In everyday life, people use a great number and variety of display artefacts: calendars, post-its, posters and pictures. Display artefacts in home environments 
have many different forms, ranging from paper displays to objects that people make visible to themselves or others. Displays serve various purposes, such as externalization of memory (reminders of actions to take, awareness of important dates), as expression of personality and social relationships (pictures of relatives, pictures of a trip, souvenirs), as media of communication to other inhabitants of the house. In our analysis we distinguish five kinds of display artefacts:

- reminder for future actions (e.g. a post-it, a shopping list);

- reminder of past events (e.g. cards of a past concert, pictures of a trip);

- awareness media (e.g. a calendar, a clock, the transportation timetable);

- communication media (e.g. a message on a board, a post-it on a door);

- $\quad$ decorative (e.g. a poster, a sculpture, a puppet).

The use of such categorization responds to the goal of understanding why and how people tend to use some kind of displays rather then others. Understanding their choices can help to identify contexts where technology and additional information makes sense in the house. The idea is not to substitute existing displays or translating them into digital formats, but rather to augment and support display based activities and to generate ideas for novel display appliances. For this reason the involvement of potential users in the design activity and their engagement with physical prototypes in a contextualized situation (in the familiarity of their own homes), becomes essential for the purpose to let people figure out possible scenarios of domestic ubiquitous computing.

\section{Related work}

As our contribution is twofold on methodology and on displays in the home we present work that is related to both areas. However, much work that is related to displays also reflects on methodologies used by the researchers.

\subsection{Approaches to the design of Ubicomp applications}

Ubicomp is inherently connected to applications and hence research in the area is most often related to application domains or specific applications. Weiser's initial work in Ubicomp [29] research can be categorized as technology driven. Systems were developed and built around a vision of available (or soon to be available) technologies. One particular focus was on solving hard engineering problems. The method of technology-driven research has been continued till today and has produced many interesting prototypes and products (e.g., in handheld computing and mobile communication). Looking at intelligent home installations (e.g. [14], [16], [19], [21], [23]) this technology driven approach can be observed too. Solving the engineering problems, developing useful system architectures and making applications robust and usable is often a first step before pushing new technologies further into everyday life. However when concerned with complex multi-purpose environments, such as the home, a pure technology driven approach is not enough as it does not consider how people like to organize their real lives. In contrast to work environments where arguments can be made on productivity and efficiency these metrics do only apply partly in people's homes. Many other issues related to aesthetics, style, personal likes and dislikes play a major role. Additionally, as people often share the environment where they live (e.g., shared houses, families, couples) it is also a collaborative environment where certain rules and roles have been established.

The method of ethnographic studies for domestic environments is discussed in [10]. In the context of IT, ethnomethodology (or ethnography, as the approach is usually referred to) has been traditionally applied in the CSCW research area to inform the design of solutions for the social organization of work. It is a method of the social science dealing with the recognition and analysis of workflows, communication, and layout of social spaces, adopted to elicit requirements for the development of new computing systems. Early applications of such methodology in the domestic environment [20] suggest that "small integrated computational appliances, supporting multiple collocated users throughout the home, is a more appropriate domestic technology than the monolithic PC'. Moreover ethnographic studies reveal that people do not live in random settings and that people have a personal understanding of how to organize their home environments. They facilitate this for collaboration and communication in their daily lives [9].

A different approach to researching the home is given by cultural probes [13]. This methodology was developed and applied in the discipline of design research, and is based on the use of open artefacts that explore the aesthetic and cultural implications of technology in everyday life. Such an approach is therefore not meant to the collection and analysis of precise data for the elicitation of user requirements; it 
rather relies on the subjective interpretation of users' expression of preferences and ideas, and is meant to gain an inspiration about how to design for new experiences.

Bringing new technology into an established home setting is not trivial. In [3] researchers investigated how people would install sensors in their environments. One finding of their research was that people cared very much about their established aesthetics of their environments, as this reflects their personalities (for themselves and even more for visitors). One lesson to be learned is that people need to participate in the creation/control of technology for the home so as to minimize the risk of building a system that nobody would ever use.

\subsection{Displays at Home}

The social construction of displays has been the focus of ethnographic studies [9], that mostly concentrate on what we call "communication media", i.e. displays that are used to communicate with one another and coordinate actions (named "coordinate displays", in [8]). In analogy to this work, Harper and Shatwell investigate the interactional properties of paper mail in the households, sustaining its affordances and important impact on the social patterns [17]. Our investigation embraces also other kinds of displays, such as decorative and awareness ones.

Perry and O'Hara have investigated the use of displays in the workplace [22]. In comparison to the working place, the domestic walls reflect much more closely the lifestyle of the people inhabiting them and become an expression of their personality and of intimacy. The decision of what to hang where, what to make easily visible for themselves and what to display for others, is highly dependent on the way people deal with information and affective media. This suggests that the location of embedded technology needs to rely on the social and spatial patterns of the house. Furthermore, the aesthetic appearance of such media and the relation to them (whether they are gifts, or memories) affects people's choices of where to put them. This implies that the design of home appliances needs to cope with the challenge to suit different people's aesthetic values and information management.

Our approach is more concrete in terms of novel technologies. Our aim is to find specific use of technologies that are compatible with everyday life and welcome in a domestic environment. We extend the methods used by technology probes [[18]] that engage people with new display artefacts.

\section{Researching domestic environments: Tools and methodology}

For this study we adopted a multi-techniques investigation which combines the methods of contextual inquiry [2], cultural probes [13], technology probes [18], scenarios-based participatory design and interviews in a qualitative research approach. This combination of techniques is extended by the use of functional technology probes to engage the user with potential solutions. The goal is to identify and interpret the attitude and the emotional aspects of users' behaviours in the house, to investigate how people think about communication in the domestic environment, what motivates, drives and pleases them.

In summary the following steps are followed:

- Step 1: Technology research, researchers have to get an understanding of potential technologies, their advantages and limitations as well as typical application areas;

- Step 2: Interview in the home environment, getting explanations why people organize their environment in the way they do it; describing the environment systematically with respect to the technologies investigated;

- Step 3: Cultural probes, investigating how people deal with certain objects, spaces, places, in their domestic environment. The focus is on artefacts that relate to the technologies that are investigated;

- Step 4: Technology probes, inspiring discussion by presenting working technology prototypes in the situation of the users home;

- Step 5: Educate the user on technologies, give the user a quick and easy to understand overview of potential technologies that are available. This needs to be in the language of the user and should also communicate pros and cons, as well as trade-offs of certain technologies;

- Step 6: Participatory design session, sketch and design a specific persona focused technology the users would like to have for their environment

- Step 7: Creating prototypes from person inspired designs, identifying generic technology artefacts or platforms for the home based on the specific user driven sketches from step 6 .

In the remainder of this section the individual steps are discussed with respect to the experiment that was carried out. Here we show exemplarily in the domain of displays for the home how this process was applied. 

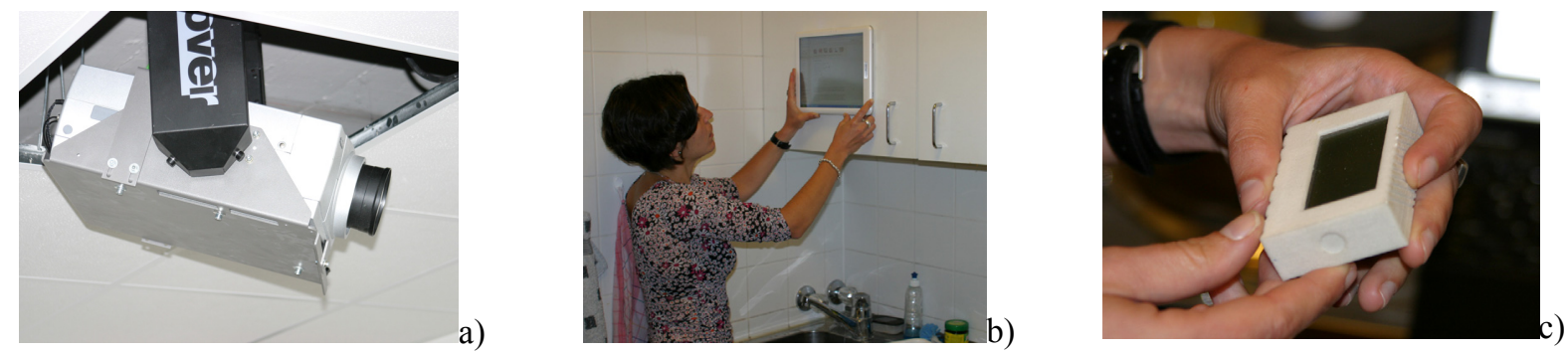

Fig. 1. Different types and technologies of displays are investigated. Figure a) shows a steerable projector for projection based interfaces; b) shows a tablet PC with touch screen technology embedded in the kitchen environment; c) shows a small wireless connected LCD display. Such displays provide different potential solutions and affordances for domestic displays.

\subsection{Technology research}

Before stating the research of potential uses of displays in a domestic context, we made an attempt to understand a wide variety of alternative display technologies. Knowing what types of technologies are available, what constraints are inherent to a technology, and how technologies can be embedded became central when discussing possible uses of displays in an environment. In particular we looked into projection based displays (steerable projection), Thin Film Transistor (TFT)-displays of various sizes, small Liquid Crystal Display (LCD) and monochrome displays and minimalist information displays (e.g., Light Emitting Diode, LED, or light as a reminder), see Fig. 1. Additionally, we researched the current state and development for interacting with displays, such as touch-screens, camera-based interaction, gestures and various sensor-based input mechanisms. We extended our technology research also to auditory displays and ambient displays.

Overall, we looked at what is commercially available, what has been suggested and demonstrated in the research community [11]. Building prototypes based on different technologies in our lab and experiencing these technologies was essential to understand them and get a good insight into possible limitations.

\subsection{Contextual inquiries}

We conducted in-depth interviews with individuals at their homes. More details concerning the sample of users and format of the interviews are provided in Section 4. The interviews took place according to a pre-defined format encompassing different phases, which provided a coherent framework for comparison of results, but still allowed freedom for brainstorming together with the interviewees. In a first step an exploratory inquiry of the household and of its display environment in particular took place. In this phase we were guided through the rooms and took pictures of display artefacts which we noticed on different surfaces and inquired about their purpose.

This inquiry is the basis to map which context could

be augmented with what type of embedded technology for different people. For each artefact we discussed with the users about the motivation of their location, the lifetime of such displays, whether they would be moved in the house, taken along outside of the house, or would be eventually thrown away. Additionally we posted questions concerning the wish to update, replicate or remotely access this kind of information. In figure 2 different kind of domestic displays are shown.

\subsection{Cultural Probes}

In a second phase, the interviewees were confronted with physical objects, which we contextualized in a brief narrative: several postcards, a very small picture $(3 \mathrm{~cm} \times 4 \mathrm{~cm})$ of a past social event, a reminder to pay a bill, a picture sent by someone met on vacation (with and without frame), a fluffy rabbit and a snowball. While investigating where people hold certain kind of information and in particular whether it belongs to private spheres (or, as Goffman describes, "back regions" [15]) or rather to public ones ("front regions") our questions also aimed at identifying what factors mostly affect this placement: e.g., the relationship to the sender, the aesthetics of the artefact, its size, its quality. We also asked users whether they would like to replicate these artefacts, edit them, scale them, or reproduce them in digital format.

\subsection{Technology Probes}

The introduction of technology probes was motivated by obstacles that we encountered in our previous research when discussing with people 
potential uses of technology. Such a methodology has already been used in [18] in a similar domain, in order to stimulate people's creativity and inspire ideas for new technology in the domestic environment. In comparison to such work, our probes were less finalized in terms of casing and hardware design, as they were meant to show the possible functionalities. This choice was motivated by the intention to avoid people's concentration on the look of the probe, but rather to stimulate their imagination in terms of scenarios of use of such technology. By presenting unfinished but working prototypes we felt that users had no hesitation when suggesting radical new form factors, usage scenarios, and applications. This is in line with the findings for prototyping in graphical user interfaces [6].

We realized that it can be very hard for people, especially when they do not have a technical background, to engage with abstract descriptions of technologies. The reactions we got can be grouped in two main categories:

- "I am happy with what I have."

People were reluctant to engage with the idea of novel technologies in their immediate environment. To them, it seemed too abstract and too distant to make an impact in their daily lives.

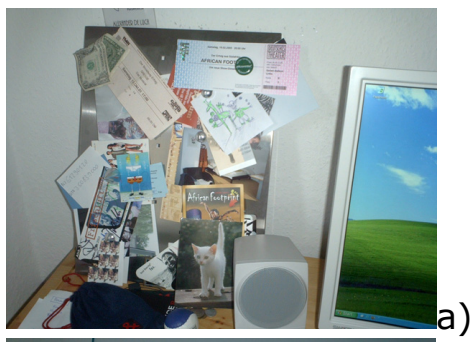

a)
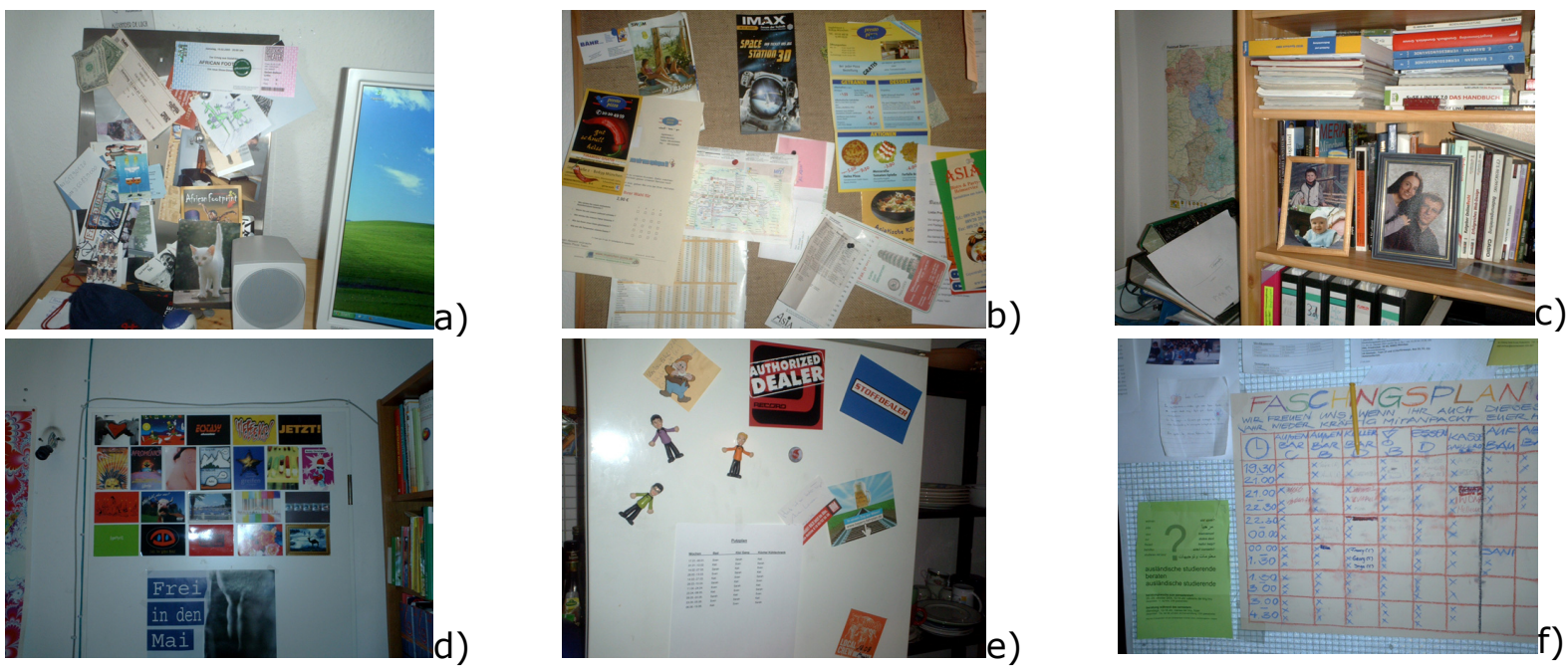

Fig. 2. Different display artefacts were discussed and documented in the visited homes. It is to be noted that people embed such artefacts in their physical environment: e.g., on the door as shown in picture d); on the fridge as shown in picture e); on the bookshelf as shown in picture c). Even though the location of such artefacts corresponds to individual spatial semantics, displays are mostly hybrid; i.e., in the same area decorative, reminder and communication displays can be found. E.g., picture f) shows a communication display that serves also the purpose of reminding scheduled activities; picture a) shows decorative display artefacts, such as the picture of the cat, together with reminders such as a concert ticket. 


\section{5. Educating the user}

Beyond the technology probes, we gave a quick overview of potential further technologies for the home. They were encouraged to imagine what kind of information could be displayed and dynamically updated. Additionally we suggested that they should imagine applications if they had an extended number of such displays, 20 or more.

With the use of technology probes and with the additional explanation which we provided we wanted to show people the following issues:

- displays can be of any size and resolution;

- displays artefacts can be wirelessly connected;

- displays artefacts can detect and communicate interaction;

- displays can be embedded into various objects and environments of everyday life.

We used simple applications or application examples for demonstrating the functionality of the hardware in a very easy-to-understand way. All our participants understood the concept of the application. It is indeed essential for the technology probes that participants understand them easily.

\subsection{Participatory design sessions}

The involvement of potential users in the design of domestic appliances was motivated by two main factors:

- Domestic environments are a very personal and private domain, in which external assumptions of technology acceptance and use might result erroneous. Getting users' insights and ideas of domestic appliances would allow us to gather a picture of how certain people envision technology in their environments;

- The way people decorate their households and handle communication in the home is very diverse, thus suggesting that different people have different needs: in order to get a heterogeneous spectrum of design alternatives, the involvement of different individuals facilitates the design of different appliances.

In the participatory design sessions people were stimulated to think about possible contexts of displays. They were asked what kind of information they would like to have where and when. In particular, in four different scenarios, that belong to four different regions of the house and are mapped to different routine activities:

- alarm clock display (e.g., when waking up or going to sleep);
- bathroom mirror display (e.g., while brushing their teeth);

- $\quad$ kitchen displays (e.g., while cooking);

- wardrobe display (e.g., when choosing clothes).

The open informal discussions were meant to collect ideas as well as to get an overall picture of the individuals and their environments. By starting with very specific display settings, people could envision such displays very easily and express their excitement or concerns. This was done so as to identify some persona, i.e., sort of user profile, that we would develop a design for.

\subsection{Creating prototypes from persona-inspired designs}

Designs for an individual provide an interesting insight in what individuals would like to have. However it seems economically not yet realistic to build individual information appliances that suit exactly one person. When creating prototypes we looked in particular for commonalities between design ideas, or for connections between individual designs. The aim when creating prototypes was to find abstractions that are valid across a set of individual designs in a particular environment and hence to understand the properties of potential appliance platforms. For each of the individual design ideas, we analyzed a potential technical realization and identified the following issues:

- potential hardware platforms (processing, communication and interaction);

- potential system software required;

- content provided and information handled.

These results provided us with a basis for creating prototypes of novel display artefacts that could accommodate the needs of various scenarios.

\section{Experiment set-up}

The interviews were conducted by two researchers in interviewees' homes and would normally take place around the kitchen table. A typical interview was scheduled to last about $60-80$ minutes. The session would start with a brief introduction to the aim of the research and to the envisioned pervasive availability of displays into everyday life. In the initial explorative phase, existing display artefacts were observed in the environments, according to our display taxonomy (see Section 1.1.): we took pictures of such displays and formulated questions regarding motivation, lifetime and personal meaning related to them. The interviewers would take notes of interviewees' answers 
and ideas, by filling a prepared questionnaire that would serve as track. The track consists of the following different phases, according to our methodological approach as outlined above:

- introduction of the study;

- $\quad$ exploratory phase of display artefacts;

- presentation of the cultural probes and related questions;

- $\quad$ introduction of the vision of display technology in everyday life;

- $\quad$ introduction of technology probes;

- participatory design discussion about four different scenarios.

Additionally, we collected socio-demographic data and interviewed people about their time management (e.g., how they manage their appointments and what calendars they use) and their electronic communication habits (e.g., their use and archiving of SMS, MMS, email, use of present systems and instant messaging).

The interview was carried out in a major European city. In total 14 people took part, seven women and seven men in age from 23 to 44 . The participants had diverse academic and professional backgrounds (e.g., computer science student, video editor, social worker, land surveyor, physics engineer). The technology equipment and computer literacy was heterogeneous (some had DSL, most had a PC, one person did not use a computer or a mobile phone). We visited six households, three flats that were shared by two people, two flats that were shared by three people and one flat that was shared by six people.

It is implicit in such an approach that small samples of users may not be representative of the whole complexity and diversity of attitudes: neither an "average" can be extracted. Our assumption, though, is that each individual may present attitudes that are representative of a segment of population. To this respect, these results should be considered as suggestive and provocative for design, instead of definitive and valid for a general "average user" that is actually inexistent.

\section{New Ideas for Display Appliances}

The experiment provided us with many insights in how people deal with displays in their home environment. In the discussion with people we got various design ideas that would fit an individual. Some of them are described in the next sub section. Generalizing these ideas we show two prototype designs that are useful and can be customized to accommodate the persona-based design suggested.

\subsection{Persona-inspired design ideas}

The use of personas in design is an established goal-directed technique [7]: the main account of such a design approach is that a 'general user' does not exist. Rather, the detailed identification of a target audience and the distinction of different profiles can effectively support the design activity. In these terms personas describe the goals and activities of archetypal users. Our interviews allowed us to sketch some primary personas that would drive our design and prototyping activity.

Given the number of people interviewed, we collected a large list of persona-based design ideas. The following list shows a selection of ideas for digital displays:

- A picture frame that periodically displays different postcards and a box that captures the postcards.

- A weather display, in particular for rain probability, in the hallway.

- An alarm clock that provides information about the schedule of the next day.

- A fixed display in the bathroom that shows the screen of the mobile phone.

- Dynamic news overview displayed onto the bathroom mirror.

- A mirror that remembers what one has worn on a certain date or occasion.

- A mirror that allows freezing an image to have a closer look.

- A display in a wardrobe that gives suggestions about what to wear in combination, after the user has picked one item she wants to wear.

- A display on the wardrobe that provides information on how to make a tie knot.

- A remotely accessible shared display for notices on the kitchen door.

- A display in the kitchen that can provide any information (television, web, video, chat, cooking instructions) at a spot that is always in the vicinity of the user.

For each of the design ideas we had an extended discussion with the users about what the appliance should do, what properties it should have and what properties it must not have. In particular we were interested in how people would like to see it embedded in the environment or artefact, how people would like to interact with it (implicitly or explicitly) and how much control over function and content they would like to have. 


\subsection{Towards prototypes}

The persona-inspired design ideas provided us with a selection of display artefacts people could imagine in their environments. By seeing the place where people imagine such displays and by discussing with them the interaction, we understood constraints that matter for potential users. Based on these collected ideas and on the constraints observed, we specified prototypes of display artefacts for the home. Each prototype combines input from multiple generated ideas. The goal of these prototypes is to understand potential display platforms for domestic use. In the following sections we present a selection of prototypes specified.

5.2.1. Networked alarm clock with information display. Information access without effort was crucial in many design ideas. Just accessing information without the need of explicit interaction, or accessing information which is embedded into an interaction that is done anyway seemed to be central requirements. For most people in our study the first information display they interact with in the morning is their alarm clock; similarly, for many people this is also the display they interact with when going to bed. Hence, as people already use its display function, several interviewees suggested to enhance the display capabilities of the alarm clock. Their suggestions for information content varied widely and included birthdays, personal or partners' schedule, weather forecast, news headlines and personal health information. A prototype resulting from such suggestions is described in [27].

In the user group we investigated, more than half did not share the flat with their partner. For them, sharing time-related (or ritual-relates) information was a further central concern. People reported to send SMS or to phone their partner when they go to bed, or in the morning when they get up. The information communicated was very much related to provide presence. Hence, using the alarm clock as a medium for presence communication was suggested.

Others suggested to extending this function beyond their partner to friends and family. Sharing information when going to bed and when getting up did not seem to be a concern. It was however central that this information was not automatically sensed but set by a gesture, such as switching on the alarm function in the alarm clock when retiring. On top of such information, it was suggested to visualize on the alarm clock who of your friends is already up and who is still sleeping. Users suggested that this may enhance communication as well as it may make people "closer". Collaborative functions such as "wake me up when most of my friends are up" were suggested too.
Technically, such a platform would consist of the alarm clock, added with a small general information display and communication facilities (e.g., UMTS). The customization would be on what information should be displayed and in which form presence information should be shared and to whom.

5.2.2. Enhanced Mirror. Most of our participants found that the mirror in the bathroom is an interesting place for accessing information, as it has been suggested by Philips in their ambient intelligence project [23]. People spend time there and during this time further information may be provided. One of our participants reported that he usually goes to the living room to have a quick look at the morning news on TV while brushing his teeth. In his case, having headline news somewhere on the mirror was the obvious thing he suggested. Mirrors in other rooms were also suggested to be enhanced with display technology, in particular the bedroom or wardrobe mirror. The information that people would have liked on a display embedded into (or next to) the bathroom mirror included upcoming birthdays and appointments, schedule information, different types of news, short entertainment (e.g., riddles to wake up and get the brain started) and tutorial information (e.g., how to make a specific tie knot). Across our participants it was central that, if the mirror is enhanced, its basic functionality should still be available without any effort. Additionally it was a general agreement that interaction should be minimal or it should even work without explicit interaction.

"Capture" and "display" were additional functionalities that would allow visualizing spots that would otherwise be invisible from a frontal perspective. People suggested for example that the mirror could freeze an image (e.g., of a spot behind the ear) and provide this as a still image for closer inspection. Similarly, people suggested that seeing yourself from behind (especially your hair) is difficult and hence a capture and display function would make this easier. In the context of the wardrobe mirror a similar functionality was proposed. A mirror that remembers what one was wearing at a specific day so that it would become possible to remember "what I wore last time I was out with the other person".

A technical solution that could accommodate these requirements is a mirror that includes a large display, a camera and a simple interface (people suggested gestures or audio commands; the idea of a touch screen was not welcome). Additionally the system has to have network access and the ability to store images with contextual information (e.g., person and time). 
Interestingly enough, the possible solutions envisioned by the interviewees were in line with the scenarios of Ambient Intelligence in the home presented by Philips Research in [[23]]. Several features envisioned by the users go much beyond the research presented.

\section{Lessons learned and discussion}

A main challenge in understanding users' acceptance and needs for emerging services and technologies is that users usually do not know the technological possibilities: what they is possible is limited by what works for them and their knowledge of today's technology.

\subsection{On methodology}

The presentation of physical prototypes, contextualized in the possible scenarios of everyday life activities that we suggested (e.g. "when you wake up", "when you brush your teeth"), was particularly useful for generating design ideas and for understanding the user profile. Indeed, people find it easier to relate to the task-oriented nature of the scenarios, than to the abstract and often functionoriented nature of a system specification. The combination of the two, scenarios of everyday life and tangible previews of future technology, proved to be a valuable method to stimulate their creativity. When embedding analogue and physical display artefacts in their homes, people are usually not concerned about overload of information and intrusion, in contradiction to when they deal with digital information. Using the technology probes people were less worried about technologies invading their homes and seemed to envision how to get hold of technology in a more personal way. It appeared that having a concrete example of technology reduced the fear of the unknown.

When looking at the results of qualitative research, it has to be kept in mind that these are not numerical, so they are not statistically reliable: rather, they are interpretative and therefore strongly depend on the researchers' empathy with the users. To this respect it was our concern to create an informal atmosphere, stimulating people to share with us the stories behind the artefacts in their homes, the social relationships that might justify certain displays, so that it would facilitate their creativity and their expression of personalities.

In the later interviews we added, at the end of the enquiry, a phase where we suggested ideas that were generated in previous interviews and asked people about their opinions. It was interesting how people reiterate on previous ideas and add their own requirements. For us these discussions and especially seeing a certain convergence on specific technology requirements were helpful when working towards prototypes.

We also got feedback from people we had visited in the following weeks, although we did not ask them. It seemed that some interviewees did not stop thinking of potential display technologies and came up with more ideas that they wanted to communicate to us. From this experience it could be interesting to let people have the technology probes for a few days to get more feedback on potential uses.

\subsection{On Display Artefacts in the Home}

In nearly all cases there were several ideas revealing that people found that additional display technologies could enrich and ease their daily private life. However, throughout the participants in our study it seemed that people manage their daily life very well and that there is no immediate an obvious need to improve "productivity". The augmentation with novel display artefacts was mostly meant to make the access to information and communication more engaging and playful. Our study showed that in many cases the ludic and playful element, as suggested in [12], are central to the life of people.

In our study the concern for efficiency was secondary: for most people their major issues were that it fits their lifestyles, their aesthetic values and it is compatible with their social life. Examples that are concerned with increased productivity in the home as presented in many ubiquitous computing scenarios (automated shopping list, to-do list, time and calendar management) did not have a great appeal with our participants. This is in line with studies on the economics of the house [4] reporting that entertainment appliances such as radio and television have diffused much faster than household and kitchen machines.

In all our discussions we could observe that people see a trade-off between having full control on the one side and automatic behaviour with minimal interaction on the other. Overall, it appeared that people would like to have display artefacts that require minimal interaction in normal operation, but allow full control by the user (e.g., to switch it completely off).

Besides using display artefacts for information access, social communication and sharing information were central issues. In many of these cases (e.g., sending an SMS to the boyfriend "I am going to bed") it appears that not the information itself is central, but 
rather the fact of being connected. Similarly, it seemed that there is a social function in types of information access (e.g., calling one's mother and ask her for a recipe instead of looking it up).

With relation to aesthetics, people imagined interwoven solutions, where technologies are part of their environment and not added to the environment. Overall it showed that locations in the domestic environment are central for the information and communication requirements. Locations trigger functions, both for information access and for initiation communication.

\section{Conclusion: Towards design guidelines for display artefacts}

Based on the small group that we studied, we concluded a number of design guidelines that can help to create novel display artefacts for domestic environments.

\subsection{Embedding information where and when it is useful}

It is central to provide the information so that the user can benefit from it. Most conventional displays we saw serve that purpose. Often the advantage is minimal (e.g., it is only useful once a month) but without providing an advantage it would not be there. We discriminate two basic functions of embedded information: to inform and to please (in some cases both functions are combined).

Usually information to inform should be embedded at points where decisions are made, or where people have choices (e.g., at the key table when someone decides on the mode of transport). The information provided should increase people's ability to make informed choices.

Information and displays with the primary function of pleasing the user are more individual and have often the function of providing a reference for remembering some person or event. For these types of displays we found that aesthetics are as important as content and this should be reflected in the design.

As it may change where and when the provision of information is most useful, such designs should be aware of their users and of the dynamic environment. Simple contexts such as "the person is not alone" or "the person is about to leave" can enhance the usefulness of a display artefact. Making displays context-aware can offer great potential: however, from our study we recommend to use this carefully as it makes the conceptual model and "predictability" of a display artefact more complicated.

\subsection{It matters how information is embedded}

The information provided should not be forced onto people or their immediate environment. Making displays unobtrusive is a central advice. If possible, it should be embedded in such a way that gives people the information without becoming an annoyance. Besides, concepts of ambient media [14] and calm technology are an important basis for the design of embedded information displays.

One further issue is that many spaces are shared and hence not completely private. For displaying personal information this has to be taken into account. In our study we saw that this is a concern even when partners are sharing a flat. In different cases people suggested that they are happy with abstracted information to be displayed as from their context they could fill in the missing information. Here again designing contextaware display artefacts is an additional possibility.

Look and feel matters: for home appliances to be accepted they need to match with the aesthetic values, lifestyles and information management of the inhabitants of the household. To this end, appliance design for the home has to take into deep consideration tools that are typical of marketing disciplines, such as segmentation and user profiles.

\subsection{Full control but no interaction required}

In the first place this seems odd, but it appeared throughout our investigation. People would like to be in control of the technology they have in their environment, but they do not want to have the responsibility to interact with it. Implicit interaction, as suggested in [23], where the system reacts to what the user does in the real world, can be a vision to achieve this. A counter example is an SMS that is received on a mobile phone. Even if you are not interested in the SMS you have to press some buttons to get back to "normal" on the phone.

For the design of information appliances it is important that there is no action required from the user when information is provided. Such designs will therefore usually require dedicated information displays that are only used for providing a specific type of information.

\section{Acknowledgement}

The work has been conducted in the context of the research projects Embedded Interaction and Fluidum that are funded by the DFG ('Deutsche Forschungsgemeinschaft'). 


\section{References}

[1] Bell, G., Kaye, J.: Designing Technology for Domestic Spaces: A Kitchen Manifesto. Gastronomica 2(2), 2002.

[2] Beyer. H., Holzblatt, K.: Contextual Inquiry. Defining Customer-Centered Systems. Morgan Kaufmann, 1998.

[3] Beckmann, S., Consolvo, S., La Marca, A.: Some Assembly Required: Supporting End-User Sensor Installation in Domestic Ubiquitous Computing. In Proc. of UbiComp 2004.

[4] Bowden, S., Offer, A.: Household Appliances and the Use of Time: The United States and Britain since 1920s, Economic History Review, Vol. XLVLL, No.4. 1994.

[5] O'Brien , J., Rodden , T., Rouncefield, M., Hughes, J.: "At home with the technology: an ethnographic study of a set-top-box trial", ACM Transactions on ComputerHuman Interaction (TOCHI), v.6 n.3, p.282-308, Sept. 1999.

[6] van Buskirk, R., Moroney, B. W.: Extending Prototyping, IBM Systems Journal - Vol. 42, No. 4, 2003 - Ease of Use.

[7] Cooper, A.: The Inmates Are Running the Asylum. SAMS, 1999.

[8] Crabtree, A., Hemmings, T. and Rodden, T.: Social construction of displays: ecological networks and coordinate displays in Public and Situated Displays: Social and Interactional Aspects of Shared Display Technologies. Kluwer Academic Publishers, 2003.

[9] Crabtree, A., Rodden, T., Hemmings, T., Benford, S. Finding a Place for Ubicomp in the Home, in Proc. UbiComp 2003, ACM Press.

[10] Crabtree, A.: The Social Organization of Communication in Domestic Settings. In Proc. of the 2003 Conference of the International Institute of Ethnomethodology and Conversation Analysis, Manchester: IIEMCA.

[11] Ferscha, A., Kortuem, G., Krüger, A. (2004). Ubicomp 2004 Workshop on Ubiquitous Display Environments, http://ubicomp.lancs.ac.uk/workshops/ubidisplay04/

[12] Gaver, W. Designing for Homo Ludens. I3 Magazine 12, pp. 2-6, June 2002.

[13] Gaver, W., Dunne, T., Pacenti, E.: Design. Cultural probes, Interactions, 6(1), 1999.

[14] Gatech, the Aware Home website: http://www.cc.gatech.edu/fce/ahri/index.html.
[15] Goffman, E.: The Presentation of Self in Everyday Life, London Penguin, 1959.

[16] Intille, S., Larson, K., Beaudin, J. S., Munguia Tapia et al.: A living laboratory for the design and evaluation of ubiquitous computing interfaces. In Extended Abstracts if the 2005 Conference on Human Factors in Computing Systems, New York, ACM Press 2005.

[17] Harper, R., Shatwell, B.: Paper-mail in the Home of the 21st Century in Inside the Smart Home, Harper Richard Ed, 2003.

[18] Hutchinson, H., Mackay, W., et Al.: Technology Probes: Inspiring Design for and with families. In Proc. CHI 2003.

[19] Innovation Center Intelligent House Duisburg, http://www.inhaus-duisburg.de

[20] Mateas, M., Salvador, T., Scholtz, J., Sorensen, D.:Engineering Ethnography in the Home. Conference on Human Factors in Computing Systems, Vancouver, Canada, 1996.

[21] MIT House_n Website: http://www.architecture.mit.edu/house_n

[22] Perry, M., O'Hara K. Display-Based Activity in the Workplace. In Proc. INTERACT'03.

[23] Philips Homelab Website, Intelligent Personal Care Environment, http://www.research.philips.com/technologies/misc/hom elab/index.html

[24] Schmidt, A. Implicit Human Computer Interaction through Context. Personal Technologies, 4(2\&3), Springer-Verlag, pp. 191-199, June 2000.

[25] Schmidt, A., Aidoo K.A., Takaluoma A., Tuomela U., Laerhoven K. van and Velde W.van de, "Advanced Interaction in Context". HUC'99, pp. 89-101. LNCS 1707.

[26] Schmidt, A., Kranz, M., Holleis, P.: Embedded Information. In Proc. Workshop Ubiquitous Display Environments in conjunction with UbiComp 2004.

[27] Schmidt, A.: Networked Alarm Clock. Design Competition 3AD Conference, 2005.

[28] Terrenghi, L., Kronen, M., Valle, C.: Usability Requirement for Mobile Service Scenarios, in the Proc. of HCI International Conference, Las Vegas, USA, July 2005.

[29] Weiser, M.: The Computer for the 21st Century. Scientific American, Vol. 265, 1991. 